\title{
Measuring neutrino mass with radioactive ions in a storage ring
}

\author{
Mats Lindroos $^{1}$, Bob McElrath ${ }^{2}$, Christopher Orme ${ }^{1,3, a}$, Thomas Schwetz ${ }^{4}$ \\ ${ }^{1} \mathrm{AB}$ division, CERN, Geneva, Switzerland \\ ${ }^{2}$ Theory division, CERN, Geneva, Switzerland \\ ${ }^{3}$ IPPP, Department of Physics, Durham University, Durham DH1 3LE, UK \\ ${ }^{4}$ Max-Planck-Institute for Nuclear Physics, P.O. Box 103980, 69029 Heidelberg, Germany
}

Received: 18 April 2009 / Revised: 4 September 2009 / Published online: 21 October 2009

(C) Springer-Verlag / Società Italiana di Fisica 2009

\begin{abstract}
We propose a method to measure the neutrino mass kinematically using beams of ions which undergo beta decay. The idea is to tune the ion beam momentum so that in most decays, the electron is forward moving with respect to the beam, and only in decays near the endpoint is the electron moving backwards. Then, by counting the backward moving electrons one can observe the effect of neutrino mass on the beta spectrum close to the endpoint. In order to reach sensitivities for $m_{v}<0.2 \mathrm{eV}$, it is necessary to control the ion momentum with a precision better than $\delta p / p<10^{-5}$, identify suitable nuclei with low $Q$-values (in the few to ten $\mathrm{keV}$ range), and one must be able to observe at least $\mathcal{O}\left(10^{18}\right)$ decays.
\end{abstract}

PACS 14.60.Pq

\section{Introduction}

There is now convincing evidence that neutrinos have mass owing to the confirmation of neutrino oscillations. Data from atmospheric [1-4], solar [5-15], reactor [16-22] and long-baseline experiments $[23,24]$ are well described by three-flavor neutrino oscillations, with the following values for the oscillation parameters [25]:

$$
\begin{aligned}
& \left|\Delta m_{31}^{2}\right|=2.40_{-0.11}^{+0.12} \times 10^{-3} \mathrm{eV}^{2}, \\
& \sin ^{2} \theta_{23}=0.50_{-0.06}^{+0.07}, \\
& \Delta m_{21}^{2}=7.65_{-0.20}^{+0.23} \times 10^{-5} \mathrm{eV}^{2}, \\
& \sin ^{2} \theta_{12}=0.30_{-0.02}^{+0.01},
\end{aligned}
$$

where $\Delta m_{j i}^{2}=m_{j}^{2}-m_{i}^{2}$ are neutrino mass-squared differences, and $\theta_{i j}$ are the lepton mixing angles. The third mixing

\footnotetext{
a e-mail: c.d.orme@durham.ac.uk
}

angle is only bounded from above with current data:

$\sin ^{2} \theta_{13}<0.040 \quad(2 \sigma)$.

Neutrino oscillation experiments have yet to determine the sign of $\Delta m_{31}^{2}$ and so we are left with two possible neutrino mass orderings. The case $\Delta m_{31}^{2}>0$ is referred to as 'normal mass ordering' while the case $\Delta m_{31}^{2}<0$ is labelled 'inverted mass ordering'. In addition, the absolute mass scale is presently unknown. If the absolute scale is small there can be a pronounced hierarchy between the masses dependent on the ordering. In particular we can have

1. Normal mass hierarchy $(N H): m_{1} \ll m_{2} \ll m_{3}$.

2. Inverted mass hierarchy $(I H): m_{3} \ll m_{1} \lesssim m_{2}$.

If the absolute scale is large compared to the mass squared splittings, the spectrum is referred to as 'quasi-degenerate' $(Q D)$, where $m_{1} \simeq m_{2} \simeq m_{3}=m_{0}$ with $m_{0}^{2} \gg\left|\Delta m_{31}^{2}\right|$. If the lightest neutrino mass is not negligible and of the order $\sqrt{\left|\Delta m_{31}^{2}\right|}$, then there exists a partial hierarchy.

At present there are three approaches to determining the neutrino mass scale: through the measurement of the electron energies near the endpoint of a beta-decay spectrum, measurement of the half-life in a neutrino-less double betadecay (if neutrinos are Majorana particles), and via study of cosmological data; see [26] for a review. An incomplete list of phenomenological studies of observables sensitive to absolute neutrino masses can be found in [27-33]. Both the observation of neutrino-less double beta decay and cosmology are model dependent approaches. In particular, it is not known whether neutrino-less double beta decay exists, and if were observed, there could be other mechanisms such as a $S U(2)_{W}$ triplet Higgs [34], Leptoquarks [35], and $R$-parity violation in Supersymmetry [36].

The present bound on the neutrino mass from kinematic studies of beta decay endpoints was obtained from the 
Mainz and Troitsk Tritium beta decay experiments [37-39]:

$m_{v}^{\mathrm{eff}}<2.3 \mathrm{eV}$

where $m_{v}^{\text {eff }}$ is the effective neutrino mass in beta decay (see below). The next iteration of the Tritium beta decay technologies is the KATRIN experiment, which should be able to place a limit $m_{v}^{\text {eff }}<0.2 \mathrm{eV}[40]$. However it is unlikely that these approaches will scale to lower neutrino masses. They can only make a positive identification of the neutrino mass if the hierarchy is quasi-degenerate. Rhenium calorimeters, such as proposed by the MARE collaboration [41], are also expected to achieve a limit $m_{v}^{\text {eff }}<0.2 \mathrm{eV}$.

Thus, new experimental technologies are required to reach the level of hierarchical masses. Furthermore, it will be important to verify the mass-mixing hypothesis for neutrino flavor conversion, as there are non-mass proposals which can cause neutrino mixing $[42,43]$. This means measuring the kinematic effect of not only the absolute mass but the mass differences as well. This requires precision at the $\sqrt{\Delta m_{31}^{2}} \simeq 0.05 \mathrm{eV}$, or even at $\sqrt{\Delta m_{21}^{2}} \simeq 0.009 \mathrm{eV}$ level. One new approach towards the neutrino mass, using ultracold atoms has been proposed recently in [44].

Here we present an idea to search for beta-decay spectrum endpoint distortions using radioactive ion beams. By tuning the boost factor of the ions, only electrons very close to the endpoint of the beta spectrum move in the direction opposite to the beam direction in the laboratory frame. In principle, this allows one to search for kinematic effects of a non-zero neutrino mass by counting the electrons with backward trajectories. We explore this possibility by performing preliminary sensitivity estimates. We specify the most important requirements on the setup in order to achieve sensitivities below $0.2 \mathrm{eV}$.

Radioactive ion beams are currently being considered as a possible source of neutrinos for a future long baseline neutrino oscillation experiment [45]. These "beta beams" are subject of intense phenomenological and R\&D studies. Beta beams with small ion boost factors, $\gamma \sim 10$, have been discussed in the neutrino literature for cross section and nuclear physics measurements at low energies, whilst boosts for long baseline experiments typically range $\gamma=80-650$. See $[46,47]$ for reviews and references. The setup proposed here has many aspects in common with such beta beams, though the boost factors needed in our case are very close to one: $\gamma \approx 1+Q / m_{e}$ or $v / c \sim \sqrt{2 Q / m_{e}}$, where the $Q$-value of the decay is assumed to be small compared to the electron mass: $Q \ll m_{e}$. In this work we do not propose a specific experimental scheme for the a measurement of neutrino mass. Whether such an experiment can be integrated in a "high- $\gamma$ " beta beam facility is an interesting question to be addressed in future studies.
This proposal has significant challenges that must be considered. These include the identification of ions with low $Q$ values in order to maximize the effect of the neutrino mass ( $Q$ in the range of few to ten $\mathrm{keV}$ ), as well as the required number of useful decays of order $\gtrsim 10^{18}$. Furthermore, the momentum spread of the ions in the beam has to be less than $10^{-5}$. This can be achieved either with classical cooling techniques or by exploring the use of "crystallized beam" technology [48-50].

This paper is structured as follows. In Sect. 2 we briefly review the phenomenology of the measurement of the neutrino mass using endpoint studies. In Sect. 3 we outline our experimental proposal, while in Sect. 4 we present simulations of the precision this setup can achieve, and the requirements on various ingredients to reach $m_{v}^{\text {eff }}<0.2 \mathrm{eV}$. In Sect. 5 we comment on challenges of the proposed measurement. A summary follows in Sect. 6. In Appendix we provide supplementary information on our measurement strategy.

\section{Beta decay endpoint phenomenology}

The standard approach for a direct mass measurement is the analysis of the endpoint region of a beta decay [37-39], see [51] for a review. A measurement is made by reconstructing of the electron spectrum of the decays of ion $I$,

$I \rightarrow I^{\prime}+e^{-}+\bar{v}_{e}$.

It should be noted that such a measurement is sensitive to the mass scale of the anti-neutrino which is assumed to be identical to its neutrino counterpart owing to CPT invariance, but it need not be [42]. Nuclei which decay through positron emission also have competing electron capture decay channels. Electron capture dominates for proton-rich nuclei with low $Q$-values. Positron decay is kinematically forbidden for $Q<2 m_{e}$ effectively prohibiting the measurement of the neutrino mass by this method.

The three mixing angles and CP-phase parameterize a mixing matrix, with elements $U_{\alpha i}$, which relate the neutrino mass eigenstates with the eigenstates that participate in the weak interaction. This mixing of the neutrino mass eigenstates means that the spectrum of the electrons from the decay should be considered as the incoherent sum of the spectra associated with each neutrino mass eigenstate,

$\frac{d \Gamma}{d E_{\beta}}=\sum_{i}\left|U_{e i}\right|^{2} \frac{d \Gamma_{i}}{d E_{\beta}}$,

where

$$
\begin{aligned}
\frac{d \Gamma_{i}}{d E_{\beta}}= & p_{\beta} E_{\beta}\left(E_{\max }-E_{\beta}\right) \sqrt{\left(E_{\max }-E_{\beta}\right)^{2}-m_{i}^{2}} \\
& \times F\left(Z, E_{\beta}\right) S\left(E_{\beta}\right)\left[1+\delta_{R}\left(Z, E_{R}\right)\right]
\end{aligned}
$$


are the individual electron spectra and $m_{i}$ is the mass for eigenstate $i$. A recent evaluation of the spectrum can be found in [52,53]. Here $E_{\beta}$ is the kinetic energy of the electron and $E_{\max }$ is the maximum electron kinetic energy for zero neutrino mass $m_{i}=0$ ("endpoint energy"). $E_{\max }$ is given by

$E_{\max }=\frac{M^{2}-M^{\prime 2}+m_{e}^{2}}{2 M}-m_{e} \approx Q$,

where $M$ and $M^{\prime}$ are the masses of the mother and daughter ions $I$ and $I^{\prime}$, respectively, and we define the $Q$-value to be $Q \equiv M-M^{\prime}-m_{e} \cdot{ }^{1}$ The approximate relation $E_{\max } \approx$ $Q$ holds under the assumptions $\left(M-M^{\prime}\right) / M \ll 1$ and $m_{e} \ll M$. Further, $S\left(E_{\beta}\right)$ is a form factor that contains the nuclear matrix element and constants

$S\left(E_{\beta}\right)=G_{F}^{2}\left(\frac{m_{e}^{5} c^{4}}{2 \pi^{3} \hbar^{7}}\right) \cos \theta_{c}\left|\mathcal{M}_{\beta}\left(E_{\beta}\right)\right|^{2}$.

The Fermi function $F\left(Z, E_{\beta}\right)$ describes the Coulomb interactions of the ejected particle. For nuclear radius $R=$ $1.2 A^{1 / 3}$ and the definitions $\eta=\alpha Z E_{\beta} / p_{\beta}$ and $\gamma=(1-$ $\left.(\alpha Z)^{2}\right)^{1 / 2}$, it takes the form

$$
\begin{aligned}
F\left(Z, E_{\beta}\right) & =4\left(\frac{2 p_{e} R}{\hbar}\right)^{2 \gamma-2} \exp (\pi \eta) \frac{|\Gamma(\gamma+i \eta)|^{2}}{|\Gamma(2 \gamma+1)|^{2}} \\
& \approx \frac{2 \pi \eta}{1-\exp (-2 \pi \eta)} .
\end{aligned}
$$

The Coulomb correction increases the decay rate for $\beta^{-}$decays and decreases it for $\beta^{+}$decays because the attraction to the nucleus enhances the decays with low momentum. $\delta_{R}$ is the contribution from electromagnetic radiative corrections [52] which are usually negligible.

The electron spectrum in (4) is parameterized by the 3 neutrino masses and the mixing angles $\theta_{12}, \theta_{13}$. However, for experiments with resolution worse than $\sqrt{\left|\Delta m_{31}^{2}\right|}$, we may parameterize the spectrum with a single effective mass $m_{v}^{\text {eff }}$, see e.g., [54-56]:

$m_{\nu}^{\mathrm{eff}}=\sqrt{\sum_{i}\left|U_{e i}\right|^{2} m_{i}^{2}} \simeq\left\{\begin{array}{l}m_{\mathrm{min}} \\ \sqrt{m_{\mathrm{min}}^{2}+\left|\Delta m_{31}^{2}\right|}\end{array}\right.$

$(\mathrm{NH})$,

where $m_{\min }$ is the mass of the lightest neutrino, and the approximate expressions hold up to terms of order $\sqrt{\Delta m_{21}^{2}} \sim$

\footnotetext{
${ }^{1}$ Strictly speaking the $Q$-value is defined as the mass difference of neutral atoms. Here we denote with $M$ and $M^{\prime}$ the actual masses of the ion before and after the beta decay, and therefore we explicitly include the electron mass in the expression for $Q$. This holds up to corrections of order of the binding energy of the electron.
}

$\sin \theta_{13} \sqrt{\left|\Delta m_{31}^{2}\right|} \sim 0.01 \mathrm{eV}$. Then the spectrum becomes

$$
\begin{aligned}
\frac{d \Gamma}{d E_{\beta}} \propto & p_{\beta} E_{\beta}\left(E_{\max }-E_{\beta}\right) \sqrt{\left(E_{\max }-E_{\beta}\right)^{2}-\left(m_{\nu}^{\text {eff }}\right)^{2}} \\
& \times F\left(Z, E_{\beta}\right) .
\end{aligned}
$$

In Fig. 1, we show the effective mass $m_{v}^{\text {eff }}$ as a function of the lightest neutrino mass $m_{\min }$. The behavior for normal mass ordering is shown in red whilst the blue lines are for inverted mass ordering. For a small minimum neutrino mass, the eigenstates separated by the solar splitting determine the size of the effective mass. For normal ordering, this pair is positioned at $m_{\min }$ resulting in a small effective mass. For inverted mass ordering, however, the solar pair is separated from $m_{\min }$ by the atmospheric mass splitting. The effective mass is a factor 5 larger as a consequence. For $m_{\text {min }} \gtrsim \sqrt{\Delta m_{\mathrm{atm}}^{2}}=0.049 \mathrm{eV}$, the atmospheric splitting is not dominant and the effective mass does not discriminate between the two orderings.

The goal of future absolute neutrino mass experiments is to push the sensitivity below $0.04 \mathrm{eV}$. Failure to measure the neutrino mass above this level identifies the mass hierarchy to be normal, on the assumption of neutrino mixing [57]. Failure to measure neutrino mass above $m_{v}^{\text {eff }} \sim 0.006 \mathrm{eV}$ would be inconsistent with our current understanding of neutrino mass and mixing given in (1). While reaching such sensitivities would be the ultimate goal of neutrino mass measurements, in this work we are slightly less ambitious. We have in mind experiments with sensitivities in the region $0.04 \mathrm{eV} \lesssim m_{v}^{\text {eff }} \lesssim 0.2 \mathrm{eV}$, and hence we can describe the spectrum by (10) using the effective neutrino mass $m_{v}^{\text {eff }}$.

From the expression for the spectrum in (10) it becomes clear that the effect of the neutrino mass is larger for de-

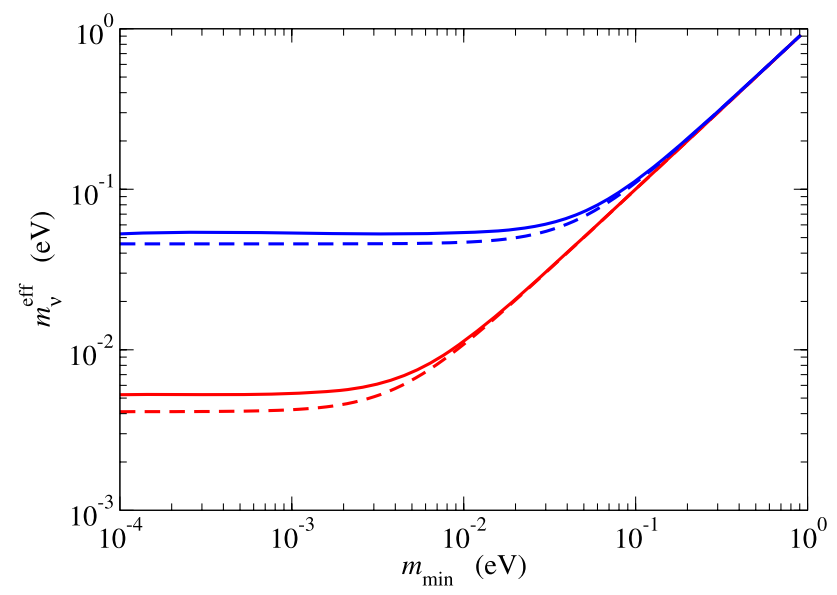

Fig. 1 (Color online) The effective mass as a function of lightest neutrino mass $m_{\min }$ for normal mass ordering (red) and inverted mass ordering (blue). In all cases, $\theta_{13}=0$. The solid lines have been simulated for the $3 \sigma$ upper bound for the solar and atmospheric parameters as defined in [25], whilst the dashed lines use the $3 \sigma$ lower bounds 
cays with a small $Q$-value. Most previous and present experiments are using Tritium, with a relatively low endpoint energy of $18.6 \mathrm{keV}$ and a half life of $12.3 \mathrm{y}$. Alternatively, the MARE project uses ${ }^{187} \mathrm{Re}$, with an even smaller endpoint of $2.47 \mathrm{keV}$, at the price of a much longer half life of 43.2 Gy. In this work we do not choose a specific ion. Instead we perform the analysis as a function of the $Q$-value. The identification of a suitable ion is central if the approach proposed in this paper is to be realized.

\section{The concept}

In this article we consider the possibility of using a very low boost ion beam as a tool to measure the neutrino mass scale, by observing backward moving electrons in the laboratory frame. A low boost $\left(v / c \sim \sqrt{2 Q / m_{e}}\right)$ radioactive ion beam is sent through an evacuated chamber with a weak magnetic field parallel to the beam line. A detector is set up on the back wall of the chamber to record the number of electrons still traveling backwards after the boost, see Fig. 2 . Therefore, the purpose of the boost is to perform a cut on the electron momenta, only selecting electrons very close to the spectrum endpoint.

Let a boosted ion have a velocity $v_{I}$ in the laboratory frame, and an electron have velocity $v_{e}$ in the rest frame of the ion. Further, let $\theta$ be the angle between $v_{e}$ and the beam direction. Hence, $v_{e}^{\|}=v_{e} \cos \theta$ is the electron velocity component parallel to the ion beam. Any electrons that satisfy $v_{I}+v_{e}^{\|}<0$ will appear in the backward direction in the laboratory frame. Let the maximum momentum of the electrons in the ion rest frame be $p_{\max }$, with

$p_{\max }=\sqrt{E_{\max }\left(E_{\max }+2 m_{e}\right)} \approx \sqrt{2 m_{e} Q}$,

where the approximation holds for small $Q$-values: $E_{\max } \approx$ $Q \ll m_{e}$. Note that in this regime, ions, as well as electrons, are non-relativistic and we can use Galilean velocity addi-

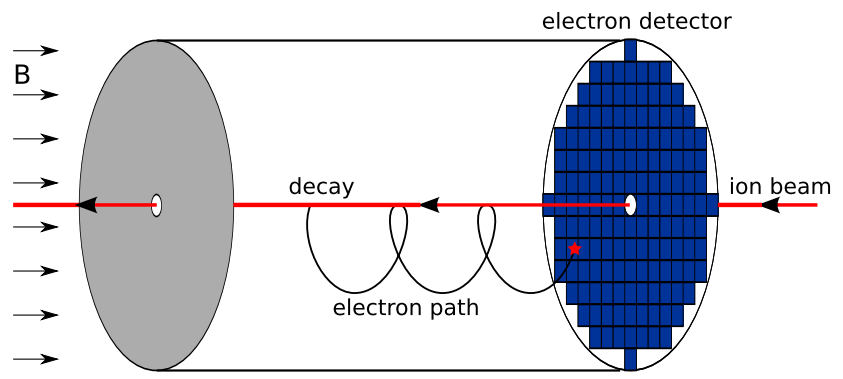

Fig. 2 Diagram of the proposed experiment. The ion beam enters an evacuated cavity whose back wall holds an electron detector. Each ejected electron follows a helical trajectory. Electrons moving in the backward direction in the laboratory frame are counted by the detector. See also Fig. 7 tion. ${ }^{2}$ Let us define an ion momentum $p_{I}^{0}$, such that an electron with momentum $p_{\max }$ emitted in the backward direction is at rest:

$\frac{p_{I}^{0}}{M}=\frac{p_{\max }}{m_{e}} \quad$ and $\quad p_{I}^{0} \approx \sqrt{\frac{2 Q}{m_{e}}} M \simeq 0.2 M\left(\frac{Q}{10 \mathrm{keV}}\right)^{1 / 2}$.

From this relation we obtain the boost factor of the ions in the beam. We have $\gamma=E_{I} / M \approx \sqrt{1+2 Q / m_{e}}$, which is close to one for the case of interest, when $Q \ll m_{e}$. Now one can alter the ion boost slightly from that value by a small $\Delta p_{I}=p_{I}^{0}-p_{I}$ such that only electrons close to the endpoint will go in the backward direction. More precisely, the ion boost will perform a cut such that only electrons with parallel momentum satisfying

$p_{\max }-\epsilon<p_{\|}<p_{\max }$

will travel in the backward direction in the laboratory frame, where from (12) we get

$\epsilon=\frac{m_{e}}{M} \Delta p_{I}$.

Hence one selects electrons with momenta close to the endpoint. Note that from (14) the cut in electron momentum $\epsilon$ is related to the change in the ion momentum by the tiny number $m_{e} / M$. Combining this relation with (11) one can translate $\epsilon$ into a cut on electron energy:

$\Delta E_{\beta} \approx \sqrt{\frac{2 Q}{m_{e}}} \epsilon=\frac{\sqrt{2 Q m_{e}}}{M} \Delta p_{I} \approx 2 Q \frac{\Delta p_{I}}{p_{I}^{0}}$.

Therefore, assuming $Q \simeq 5 \mathrm{keV}$, sensitivity to neutrino masses of order $\Delta E_{\beta} \sim 0.1 \mathrm{eV}$ requires control of the ion momentum at the level of $\delta p / p \sim 10^{-5}$.

The total number of electrons going in the backward direction can be calculated by transforming to cylindrical coordinates: $p_{\perp} d p_{\perp} d p_{\|}=2 p\left(E_{\beta}+m_{e}\right) d E_{\beta}$ with $p^{2}=p_{\perp}^{2}+p_{\|}^{2}=E_{\beta}\left(E_{\beta}+2 m_{e}\right)$. Then one finds

$$
N(\epsilon)=\frac{1}{2} \int_{p_{\max }-\epsilon}^{p_{\max }} d p_{\|} \int_{0}^{\sqrt{p_{\max }^{2}-p_{\|}^{2}}} d p_{\perp} \frac{d \Gamma}{d E_{\beta}} \frac{p_{\perp}}{p\left(E_{\beta}+m_{e}\right)} .
$$

In Fig. 3 we show the number of electrons going backward relative to the total number of electrons, which is given by

$N_{\text {tot }}=2 N\left(\epsilon=p_{\max }\right)=\int_{0}^{E_{\max }} d E_{\beta} \frac{d \Gamma}{d E_{\beta}}$.

\footnotetext{
${ }^{2}$ We have checked explicitly that relativistic effects are small and can be neglected to good approximation. Note that the neutrino is relativistic and its momentum is not Galilean invariant.
} 
Fig. 3 The fraction of electrons that travel in the backward direction (16) in the laboratory frame as a function of the cut on the electron momentum parallel to the beam, $\epsilon$, see (13). A $Q$-value of $5 \mathrm{keV}$ has been considered for a selection of neutrino masses from zero to $m_{v}=1 \mathrm{eV}$. The right panel is a zoom into the $\epsilon$ region from 2 to $10 \mathrm{eV}$
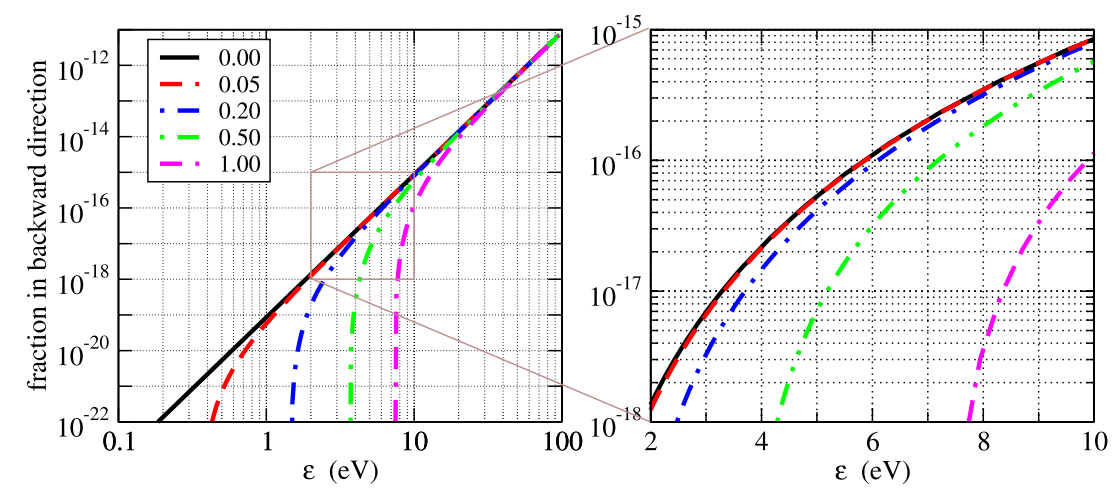

The difference between $m_{v}^{\text {eff }}=0$ and $m_{v}^{\text {eff }} \sim 0.2 \mathrm{eV}$ is at the level of $10^{-18}$ and we conclude that in order to reach these sensitivities to the neutrino masses, of order $\gtrsim 10^{18}$ ion decays are needed. This number, of course, represents a major challenge for the proposed technique. Now we assume a magnetic field $B$ parallel to the beam axis. The electrons will spiral with gyro-radius $p_{\perp} / q B(q=1$ for electrons). Hence, electrons with a given orthogonal momentum component will hit either the front or back wall of the cavern at a distance from the beam axis ranging from zero to $R_{\max }\left(p_{\perp}\right)=2 p_{\perp} / B$. The distribution of events as a function of $R$ encodes some spectral information, the use of which slightly improves our sensitivity, as described in Appendix. A traditional endpoint search, such as KATRIN uses an electric field to select electrons close to the endpoint; a cut on the momentum $p>p_{\text {cut }}$ such that a shell of the momentum sphere in the ion rest frame is selected. In our case we cut on the momentum component parallel to the beam. Hence, we use only a slice of the momentum sphere, which has the disadvantage that many electrons close to the endpoint are lost. Roughly, the fraction of electrons in the slice $\left(p_{\max }-\epsilon<p_{\|}<p_{\max }\right)$ and the shell $\left(p_{\text {cut }}=p_{\max }-\epsilon<\right.$ $\left.p<p_{\text {max }}\right)$ is given by $\left(p_{\perp}^{\max } / p_{\max }\right)^{2} \sim \epsilon / \sqrt{m_{e} Q} \sim 10^{-4}$, where $p_{\perp}^{\max } \approx \sqrt{2 p_{\max } \epsilon}$ and the value $10^{-4}$ is obtained for $\epsilon \sim 5 \mathrm{eV}$ and $Q \sim 5 \mathrm{keV}$. This small factor of useful decays has to be compensated by the total number of ion decays, which is part of the reason our proposal requires $>10^{18} \mathrm{de}$ cays.

\section{Simulations and sensitivity estimations}

\subsection{Description of the analysis}

To extract the neutrino mass from an experiment as suggested here, one would perform a measurement at many values of $\epsilon$ in order to sample the spectrum shown in Fig. 3 . Since the count rate increases very fast with $\epsilon$ one would optimize the measurement time at a given $\epsilon$ such that most time is spent close to the endpoint at small $\epsilon$, whereas the necessary time in order to accumulate enough events decreases fast with increasing $\epsilon$. For each experimental run at fixed $\epsilon$, one can perform a fit to the distribution of events as a function of $R$, the distance of the detected event from the beam axis, as discussed in Appendix. Before commenting on such a full-fledged analysis, we discuss first a simplified analysis using only the total number of counts (no radial information) at two values of $\epsilon$.

At least two data points are needed to simultaneously extract the $Q$-value of the ion. Typical uncertainties on $Q$ are too large compared to the precision required in order to use the $Q$-value as an input for the endpoint measurement [58]. For example, the $Q$-value for Tritium decay has been determined as $18589.8 \pm 1.2 \mathrm{eV}$ [59], to be compared to the $0.2 \mathrm{eV}$ sensitivity goal of KATRIN. Future high precision measurements at the MPIK/UW-PTMS in Heidelberg [60] aim at a precision for $Q$ of $30 \mathrm{meV}$, which may be used as cross check for the KATRIN experiment. In general it is therefore necessary to fit for the $Q$-value, in addition to the neutrino mass [40,58]. Even though the neutrino mass and $Q$-value are not degenerate if full spectral information is available, in an analysis of the total counts at a single $\epsilon$, the effect of a non-zero neutrino mass can mimic a slightly larger $Q$-value. Hence, a measurement at a single value for the ion boost is not sufficient to fit for the neutrino mass.

In the simulations carried out in this study, we combine two experimental runs: one with small $\epsilon$ close to the endpoint and one with large $\epsilon$. At large $\epsilon$ the total count rate is several orders of magnitude larger than the change invoked by a non-zero neutrino mass. Hence, a 2-parameter fit for the neutrino mass and $Q$-value at large $\epsilon$ will be largely independent of the neutrino mass, i.e. one effectively makes a measurement of the $Q$-value. For small $\epsilon$, however, the total count rate becomes comparable to the reduction for nonzero neutrino mass. There is a strong neutrino mass dependence in this case which, when combined with the $Q$-value measurement from the run with large $\epsilon$, constrains the neutrino mass. 
Quantitatively, in our numerical analyses we calculate the following $\chi^{2}$

$\chi^{2}=\min \left[\chi_{\epsilon_{1}}^{2}+\chi_{\epsilon_{2}}^{2}+\left(\frac{\eta}{\sigma_{\text {norm }}}\right)^{2}\right]$.

Our $\chi_{\epsilon_{i}}^{2}$ definition is based on Poisson statistics:

$\chi_{\epsilon_{i}}^{2}=2\left[N_{\epsilon_{i}}-D_{\epsilon_{i}}+D_{\epsilon_{i}} \ln \left(\frac{D_{\epsilon_{i}}}{N_{\epsilon_{i}}}\right)\right]$,

where

$N_{\epsilon_{i}}=(1+\eta) T_{\epsilon_{i}}\left(m_{v}^{\mathrm{eff}}, \Delta Q\right)$,

$D_{\epsilon_{i}}=T_{\epsilon_{i}}\left(m_{v}^{\text {true }}, \Delta Q=0\right)$.

$T_{\epsilon_{i}}\left(m_{v}^{\text {eff }}, \Delta Q\right)$ is the predicted number of events for displacement $\epsilon_{i}$ for a certain neutrino mass hypothesis $m_{v}^{\text {eff }}$ and a shift in the $Q$-value of $\Delta Q$. The pull parameter $\eta$ takes into account a systematic uncertainty on the overall normalization of the number of events, $\sigma_{\text {norm }}$, which we assume to be correlated between the two runs. $D_{\epsilon_{i}}$ is the simulated data for displacement $\epsilon_{i}$ at an assumed "true value" for $m_{v}^{\text {eff }}$ and at the "true" $Q$-value. The final $\chi^{2}$ is found by adding the penalty term $\left(\eta / \sigma_{\text {norm }}\right)^{2}$ and then minimizing with respect to the pull. Owing to the large uncertainties on the $Q$-value, we always treat $\Delta Q$ as a free parameter. We assume $100 \%$ detection efficiency. A smaller efficiency would just rescale the required total number of events accordingly and an error on the efficiency would contribute to $\sigma_{\text {norm }}$.

In Fig. 4 we demonstrate this approach for the case $Q=$ $5 \mathrm{keV}$ and $m_{v}^{\text {true }}=0.1 \mathrm{eV}$, both for no systematics and a $2 \%$ error on the normalization of the flux. Runs with $\epsilon=5 \mathrm{eV}$ and $100 \mathrm{eV}$ have been taken with measurement times in the ratio 99:1 such that the total number of useful ion decays is
$10^{18}$. It is evident from Fig. 4 that for large $\epsilon$, the fit only has a very slight dependence on the neutrino mass and provides a measurement of $Q$, where the accuracy on the $Q$-value is sensitive to uncertainty on the normalization of the number of useful decays. For small $\epsilon$, on the other hand, we see a strong dependence on the neutrino mass which shows little variation with the normalization of the flux. Obviously, a low $\epsilon$ measurement alone cannot constrain $m_{v}^{\text {eff }}$ because of the correlation with $\Delta Q$. However, the combination of small and large $\epsilon$ runs provides sensitivity to the neutrino mass. The effect of the systematics on the neutrino mass sensitivity is felt through the uncertainty on the $Q$-value.

We have checked that the two- $\epsilon$ run analysis provides already a good sensitivity. Certainly there is room for improvement by exploring many runs at different $\epsilon$ values with optimized measurement times at each position. While a detailed optimization along these lines is beyond the scope of the present work, preliminary studies indicate that the sensitivity may be improved by a factor of order $10 \%$, similar to exploring the radial information as described in Appendix.

Figure 4 indicates that the proposed measurement may provide a determination of the $Q$-value at the sub-eV level. Let us stress that this would rely on a perfect knowledge of the momentum of the ion in the beam $p_{I}$. It turns out that $\Delta Q$ is fully correlated with $p_{I}$ and what actually is measured is the sum of the two. Therefore, if we are interested mainly in a measurement of the neutrino mass, without the ambition to determine also $Q$, we do not need to know $p_{I}$ with very good accuracy, since we simply fit for the sum $p_{I}+Q$. However, it is very important that the momentum spread $\delta p_{I}$ of the beam is small enough, such that the spectral distortion introduced by $m_{v}^{\text {eff }}$ is not washed out. We are going to quantify this requirement below.

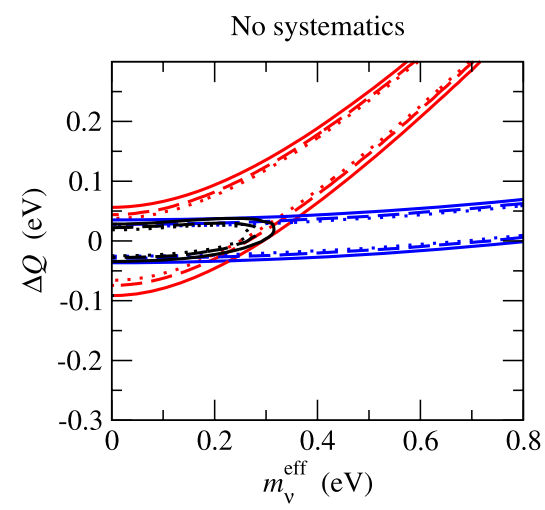

Fig. 4 We show the 90\%, 95\% and 99\% confidence level contours for two degrees of freedom. We assume a $Q$-value of $5 \mathrm{keV}, 10^{18}$ useful ion decays, and a "true" neutrino mass of $m_{v}=0.1 \mathrm{eV}$. Results are shown separately for $\epsilon=5 \mathrm{eV}$ and $\epsilon=100 \mathrm{eV}$, and their combination. The $\epsilon=100 \mathrm{eV}$ run shows little dependence on the neutrino mass
Error on flux: $2 \%$

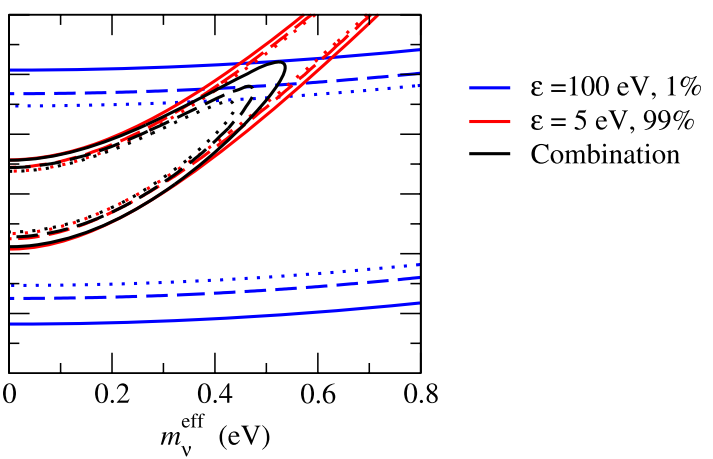

whereas for $\epsilon=5 \mathrm{eV}$ the dependence is strong. Their combination constrains the neutrino mass'. In the left panel no systematical errors are assumed, whereas in the right panel, we introduce a $2 \%$ error on the flux normalization. No backgrounds have been included in these analyses 
4.2 Requirements to reach sub- $0.2 \mathrm{eV}$ sensitivity on the neutrino mass

In Fig. 5, we present the upper bound on $m_{v}^{\text {eff }}$ at $90 \%$ confidence level, which can be obtained if the true value is $m_{v}^{\text {true }}=0$, as a function of useful decays for $Q$-values of 2, 4 and $8 \mathrm{keV}$. Runs of $\epsilon=5 \mathrm{eV}$ and $\epsilon=100 \mathrm{eV}$ are considered in the ratio 99:1 such that the total useful number of decays sum up to the value shown on the horizontal axis. No backgrounds or systematics have been included, and we neglect the momentum spread of the ions. It is seen from Fig. 5 that the approach adopted in this paper places very strong requirements on $Q$ and the luminosity. To match the expected sensitivity of the KATRIN and MARE experiments of $m_{v}^{\text {eff }}<0.2 \mathrm{eV}$, one requires $4 \times 10^{16}, 5 \times 10^{17}, 8 \times 10^{18}$ decays for $Q$-values of $2,4,8 \mathrm{keV}$, respectively. A factor 5 improvement down to $m_{v}^{\text {eff }}<0.04 \mathrm{eV}$, which will separate the normal and inverted mass hierarchy regions in Fig. 1,

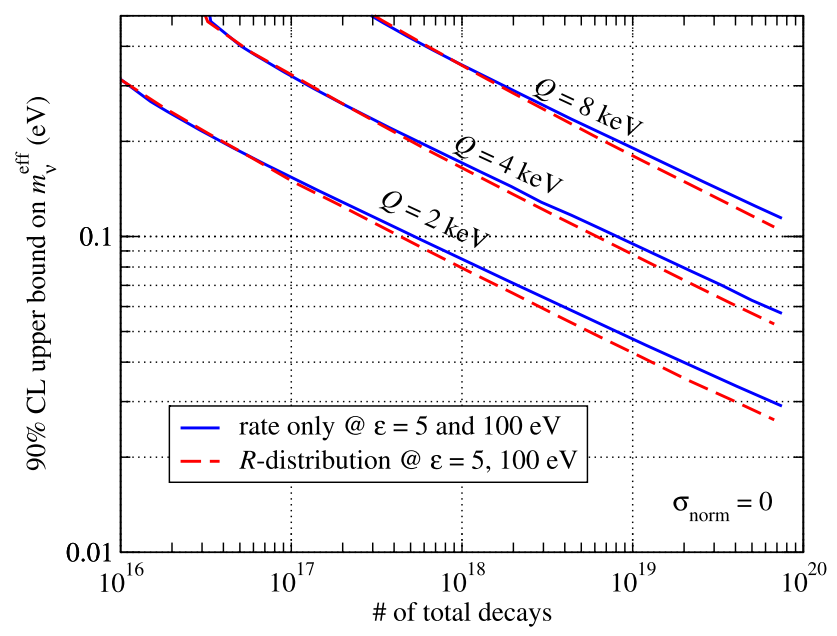

Fig. 5 Sensitivity to the neutrino mass at $90 \%$ confidence level as a function of useful decays for $Q=2,4,8 \mathrm{keV}$. We show the total rate analyses with two ion boosts corresponding $\epsilon=5$ and $100 \mathrm{eV}$ (solid) and an analysis with using also the radial distribution with 20 bins of equal width in $R$ (dashed) requires in excess of $10^{19}$ counts across the run of the experiment.

We also show in Fig. 5 an analysis that takes into account the radial distribution of the backward moving electrons, as described in Appendix. 20 bins of equal radial width were used with the same run parameters as the total rates analysis. There is only minor improvement when radial information is included in the very high luminosity region, because this approach does not reconstruct the electron energy spectrum itself, as electrons with transverse momentum $p_{\perp}$ can strike the detector at any radius less than twice their gyroradius $r_{g}=2 p_{\perp} / B$. The spectral information is thus significantly smeared out and cannot be reconstructed accurately. Although neither analysis has been rigorously optimized, one does not expect order of magnitude increases in sensitivity. Therefore, in the following, we only consider the two- $\epsilon$ run analysis described in Sect. 4.1.

In Fig. 6 we examine the behavior of neutrino mass sensitivity with systematics, backgrounds and the ion beam momentum spread. We consider the cases $Q=3 \mathrm{keV}$ with $10^{19}$ useful decays ("high sensitivity") and $Q=5 \mathrm{keV}$ with $10^{18}$ useful decays ("low sensitivity"), and, as before, in both cases we run at $\epsilon=5$ and $100 \mathrm{eV}$ with a ratio of 99:1. The "low" and "high" sensitivity configurations provide nominal sensitivities of 0.21 and $0.071 \mathrm{eV}$, respectively. The numbers of events obtained for these configurations are given in Table 1 . Now we include each one of the three above mentioned effects separately, in order to investigate at which level the sensitivity starts to deviate from these idealized numbers.

From the left panel of Fig. 6, it is seen that the sensitivity starts to deteriorate rapidly for normalization uncertainties greater than $1 \%$. This can be understood in conjunction with Fig. 4 where it was shown how the error on the normalization on the number of useful decays affects the overall sensitivity. The systematics severely limits the ability of the $\epsilon=100 \mathrm{eV}$ run to constrain the $Q$-value which in turn worsens the sensitivity to the neutrino mass. This is true for both cases considered, but especially the high sensitivity setup which becomes independent of $\sigma_{\text {norm }}$ only below
Fig. 6 Sensitivity to $m_{v}^{\text {eff }}$ at $90 \%$ confidence level for $Q=5 \mathrm{keV}$ and $10^{18}$ useful decays (dashed/blue) and $Q=3 \mathrm{keV}$ and $10^{19}$ useful decays (solid/red). In the left panel, the effect of the normalization uncertainty on the flux is considered; background levels are varied in the center panel; whilst the effect of the momentum spread of the initial ion is taken into account in the right panel

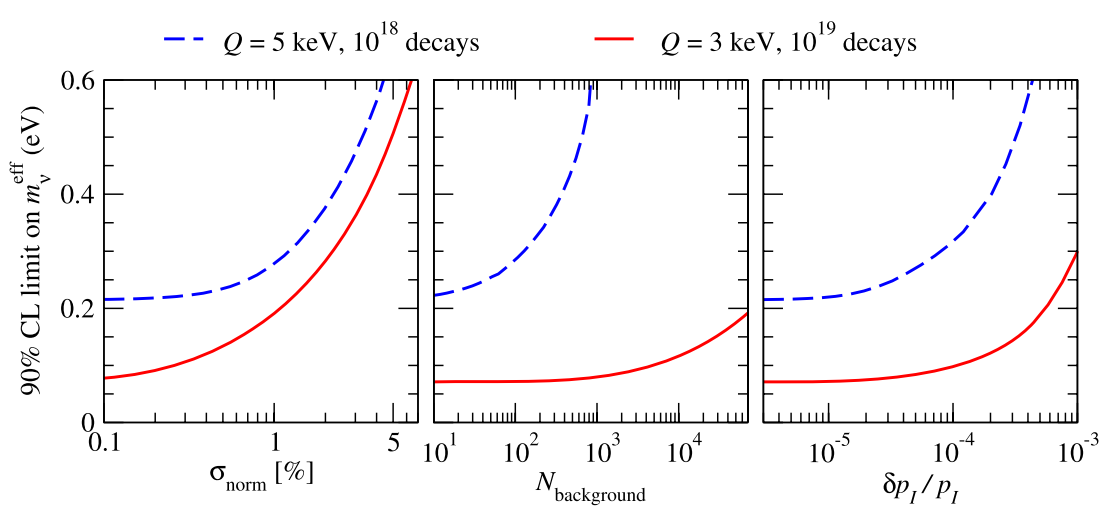


Table 1 Number of events obtained for the "low" and "high" sensitivity configurations defined in the text. For $\epsilon=5 \mathrm{eV}$ we show numbers for $m_{v}^{\text {eff }}=0$ and $0.2 \mathrm{eV}$, whereas for $\epsilon=100 \mathrm{eV}$ event numbers are

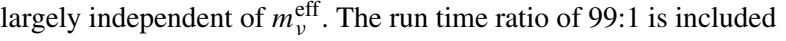

\begin{tabular}{ccc}
$\frac{\epsilon=5 \mathrm{eV}}{m_{v}^{\text {eff }}=0}$ & $m_{v}^{\text {eff }}=0.2 \mathrm{eV}$ & $\epsilon=100 \mathrm{eV}$ \\
51 & 40 & $8.2 \times 10^{4}$ \\
1440 & 946 & $2.3 \times 10^{6}$ \\
\hline
\end{tabular}

$0.1 \%$. Naively such an accuracy seems not very difficult to achieve. Apart from traditional beam flux determinations, one could measure the electrons emitted in the forward direction by installing an electron detector at the front wall of the chamber. The $\mathcal{O}\left(10^{18}\right)$ electrons may provide a means to determine the total number of decays with negligible statistical error.

In the center panel of Fig. 6, the effect of including backgrounds is shown. We assume that the magnetic field is adjusted such that the total area illuminated by the electrons on the back wall is the same for both the small and large $\epsilon$. A constant background rate is taken. The number of background events shown on the horizontal axis is the combined background from both runs. Here, the behavior is as one would expect; the low sensitivity setup suffers badly with loss in sensitivity even for background rates less than 100 across the entirety of the experiment. The high sensitivity setup performs much better; it is able to tolerate up to 1000 background events before the sensitivity starts to diminish.

In the proposed experiment there are several potentially dangerous sources of backgrounds. Even a tiny fraction of the $10^{18}$ to $10^{19}$ electrons supposed to go in the forward direction, which could be deflected by some effect to the back wall, would constitute a serious background for the measurement. Note that the forward-going decay electrons are high energy, and can eject electrons from the forward wall. These could then spiral backwards and hit the rear wall. This could be mitigated somewhat by removing the magnetic field in the forward direction, and using timing information of the beam bunch relative to the electron detection. We also ignore the electric and magnetic field generated by the beam, which will affect the trajectory of the ejected electrons. Beam-related backgrounds can be measured by adjusting the ion's momentum so that $\epsilon<0$ and no electrons should have a backward trajectory, while the beam-unrelated background can be determined from beam-off periods.

In the right panel of Fig. 6, we present the sensitivity as a function of the spread of the ion momentum in the beam direction. We assume a Gaussian momentum distribution in the beam direction with width $\delta p_{I}$. From (15), this translates into a spread on $\epsilon$ of

$\delta \epsilon=\sqrt{2 Q m_{e}} \frac{\delta p_{I}}{p_{I}}$.
In order to include this uncertainty in the analysis we fold the number of events $N(\epsilon)$, given in (16), with a Gaussian with width $\delta \epsilon$. This procedure takes into account the momentum spread parallel to the beam, whereas the momentum uncertainty perpendicular to the beam is not included. This will be important in reality, especially in a setup that uses the radial distribution of electrons, which, however, has only a minor impact on the sensitivity, cf. Fig. 5.

In the right panel of Fig. 6 one can see that for the low sensitivity setup, the sensitivity to the neutrino mass starts to abate at $\delta p_{I} / p_{I} \sim 2 \times 10^{-5}$. This is consistent with the relation between the uncertainty on the electron energy and the ion momentum spread:

$\delta E_{\beta}=2 E_{\beta} \frac{\delta p_{I}}{p_{I}}+\mathcal{O}\left[\left(\frac{E_{\beta}}{m_{e}}\right)^{2}\right]$.

For $\delta E_{\beta}=0.22 \mathrm{eV}$ and $Q=5 \mathrm{keV}$, one requires an uncertainty on the momentum to be $\delta p_{I} / p_{I}<2.2 \times 10^{-5}$ for $E_{\beta} \approx Q$. The same estimate for the high sensitivity setup predicts $\delta E_{\beta} \sim 0.075 \mathrm{eV}$ for $\delta p_{I} / p_{I} \sim 1.2 \times 10^{-5}$. However, from Fig. 6 we observe that the sensitivity starts to deteriorate only at $\delta p_{I} / p_{I} \sim 1.0 \times 10^{-4}$ where, according to (22), $\delta E_{\beta} \sim 0.6 \mathrm{eV}$. This behaviour indicates that the higher count rates can compensate for less precision on the ion beam momentum. In summary, to reach interesting sensitivities to the neutrino mass, momentum spreads in the range $10^{-5}$ to $10^{-4}$ are required.

\section{Challenges and technology requirements}

The goal of this work is not to propose a realistic experimental setup but just to present the basic idea towards using ion beams for a neutrino mass measurement. Nevertheless we add in this section a few comments on the experimental configuration suggested and various challenges of our proposal, without the ambition of being complete.

\subsection{Comments on the experimental configuration}

As we have discussed above, the main information on the neutrino mass comes from the total number of events, whereas the $R$ distribution of events (as a function of the distance from the beam axis) leads to a very small extra gain in sensitivity, cf. Fig. 5. Therefore, the spacial resolution of the electron detector is not crucial. In particular, the typical granularity of silicon detectors is about 1 micron which can be chosen to be much smaller than the bin size in $R$ by adjusting the magnetic field strength.

The main requirements on the $B$-field are that it should be parallel to the beam axis with very good precision. Any $B$-field component perpendicular to the beam may deflect 
forward going electrons. A $B$-field fluctuation in this direction should have a similar effect as the $\delta p_{I} / p_{I}$ uncertainty discussed in the previous section. An inhomogeneity in the direction parallel to the beam should have a minor impact on the result, since this would lead just to a smearing of the radial distribution, which does not impact much the sensitivity. The $g-2$ collaboration has achieved a precision on the magnetic field homogeneity of $10^{-6}$ [61]. While their field is much stronger than required here, we note that there is some flexibility on the required field strength, which is basically determined by the feasible size of the cavern.

Residual gas particles in the cavern may lead to a background, since electrons or beam ions may scatter off them and lead to some additional event rate at the detector. The required quality of the vacuum has to be estimated to reach the background levels discussed in the previous section. Furthermore, the forward going electrons hitting the wall of the cavern might kick out particles from the surface, an effect with must not deteriorate the vacuum beyond the acceptable level.

\subsection{Total number of decays}

Our analysis indicates that we would require $10^{18}-10^{20}$ useful decays to reach sub-KATRIN sensitivities. Such production is in line with projected EURISOL intensities [62-64]. However, to store such a large number of ions is a major challenge, especially as they will be long lived and possibly have high charge. Space charge effects, the defocussing of the beam due to beam-particle interactions, will be significant at non-relativistic velocities.

\subsection{Separating forward and backward moving electrons}

A major challenge of the proposed experiment will be the separation of forward and backward moving electrons. Since there will be of order $10^{16}$ more electrons moving forward than backward, the separation has to be better than $10^{-16}$. At that level one can imagine many dangerous effects which may lead to a background. For example, forward moving electrons will create backward moving electrons by collisions with the walls of the cavern or interactions with residual gas. Furthermore, any electric field parallel to the beam, or a magnetic field component perpendicular to the beam should be avoided. A suitable experimental configuration to overcome this challenge must be identified.

\subsection{Momentum spread requirements and coulomb crystals in storage rings}

The mass measurements discussed in this paper can only be realized if the energy spread of the ions in the ring is very small. For an electron with kinetic energy $E_{\beta}$, an uncertainty on the ion momentum $\delta p_{I} / p_{I}$ translates to an uncertainty on the electron energy via (22). For example, an endpoint electron from an ion with $Q=5 \mathrm{keV}$, an uncertainty on its kinetic energy of $0.1 \mathrm{eV}$ requires $\delta p_{I} / p_{I}=1 \times 10^{-5}$. The numerical results presented in Sect. 4.2 indicate that in order to reach interesting sensitivities to the neutrino mass, ion momentum spreads in the range $10^{-5}$ to $10^{-4}$ are required. Values in this range may be achieved with classical beam cooling for low energy ion beams with electron cooling or laser cooling. Therefore, if ions with very low $Q$-values $(\mathcal{O}(1 \mathrm{keV}))$ are available, classical cooling is sufficient. Availability of such low $Q$-values in nature is minimal, however, those that do exist are not practical. For example, ${ }^{187} \operatorname{Re}$ has $Q=2.6 \mathrm{keV}$, but it also has a half-life of $4.5 \times 10^{10}$ years. New coolings techniques will be required for the likely much larger $Q$-values.

For cooled low intensity ion beams of 5000-10000 ions, a distinct transition to much lower momentum spread has been observed with increasing electron cooling current at NAP-M in Novosibirsk [65, 66], ESR at GSI [67] and CRYRING at MSL [68]. This has been interpreted as an ordering of the ions in a regime where the energy spread of the beam is too small to permit any individual ion to overtake or drop behind its neighbors. Effectively, the ions will create a one dimensional string of ions with a minimum distance separating individual ions. This distance is defined classically by setting kinetic and potential energy equal, giving $d_{\min }=(Z q)^{2} / 4 \pi \epsilon_{0} k T$ where $k T$ is the ion temperature [68]. The momentum spread is measured to be smaller than $10^{-6}$ with the upper limit given by the known ripple of the power supplies controlling the electromagnetic fields which confine the beam. The ultimate momentum spread is set by the cooling forces acting on the beam. First studies have shown that bunching of ordered beam should be possible enabling at least moderate acceleration [69]. To create much higher energy ordered beams it is necessary to develop cooling schemes for highly relativistic beams as the ordered beam bunch is unlikely to survive transition. Development of electron cooling schemes for ultra relativistic ion beams are underway at BNL [70], for example. The new HEstorage rings at the FAIR facility in Germany will in time enable experiments with laser cooling of highly relativistic beams [71].

For the low energy regime, experimental work is in progress in Japan at the S-LSR ring in Kyoto [72]. They have demonstrated one dimensional ordering of protons [73] and are working on special tapered cooling schemes in a dispersion free storage ring for experiments aiming to achieve three dimensional ordering of highly charged ions.

Potential new applications such as the one proposed in this paper could motivate R\&D on ordered beams. Such efforts could eventually yield higher intensity and/or higher energy ordered beams. Studies are needed on increasing 
the number of ions, and ensuring the desired properties of the beam at high densities. All experiments on crystallized beams carried out thus far [50] have used less than 10000 ions.

\subsection{Comments on $Q$-values}

In this paper, we have established the necessity of $>10^{18}$ decays and $Q$-values $\sim 1-10 \mathrm{keV}$ if accelerated ions are to be used to measure the neutrino mass at sub-KATRIN levels. This measurement therefore needs a low $Q$-value ion with a short half-life. Rhenium- 187 possesses the smallest $Q$-value known: $2.6 \mathrm{KeV}$; however, the $5 / 2^{+} \rightarrow 1 / 2^{-}$transition has a half-life of $4.5 \times 10^{10} \mathrm{y}$. For the set of beta emitting nuclei with half-lives $<10 \mathrm{y}$, Ruthenium-106 and Radium-228 have the smallest $Q$-values: $39.4 \mathrm{keV}$ and $45.9 \mathrm{keV}$, respectively. The total number of decays will need to be increased by 3-4 orders of magnitude to match the sensitivities discussed in Sect. 4.2 if these ions were to be used.

The most promising ion is therefore Tritium with $Q=$ $18.6 \mathrm{keV}$, however electron cooling is necessary rather than laser cooling because it has no hyperfine structure when ionized. Tritium has the added advantage that the number of ions that can be obtained is orders of magnitude larger than those discussed in this paper, as it is naturally occurring and does not need to be produced in an accelerator.

\section{Summary and conclusions}

We have discussed the possibility to use radioactive ion beams to study the kinematic effects of a non-zero neutrino mass close to the endpoint of the beta decay spectrum. The underlying idea is to adjust the boost factor of the ions in such a way that only electrons close to the endpoint will have backward trajectories in the laboratory frame. We have discussed some requirements of the proposed setup in order to exceed the sensitivity of $0.2 \mathrm{eV}$ of the latest generation of Tritium and Rhenium decay experiments. A crucial question is whether it will be possible to accelerate enough ions within reasonable time such that of order $10^{18}-10^{20}$ decays can be observed. This issue is also related to the identification of a suitable ion with a low enough $Q$-value (in order to maximize the effect of the neutrino mass), and a small enough half life (in order to have a high enough decay rate).

As an example, for a very low $Q$-value of $2 \mathrm{keV}$, one needs $4 \times 10^{16}$ decays to obtain the KATRIN sensitivity of $0.2 \mathrm{eV}$, while $10^{19}$ decays will allow for a $m_{v}^{\text {eff }}$ measurement at $0.04 \mathrm{eV}$. On the other hand, if no suitable ion with such a low $Q$-value can be identified the requirements on the total number of decays increases drastically: for $Q=4(8) \mathrm{keV}$ the $0.2 \mathrm{eV}$ sensitivity is reached for $5 \times 10^{17}\left(8 \times 10^{18}\right) \mathrm{de}-$ cays, respectively. The sensitivity goal of $m_{v}^{\text {eff }}<0.04 \mathrm{eV}$, which will separate the normal and inverted neutrino mass hierarchy regions, requires in excess of $10^{19}$ counts across the run of the experiment together with a $Q$-value of $2 \mathrm{keV}$.

Our proposal relies on a very small momentum spread of the ions, at the level of $\delta p_{I} / p_{I} \lesssim 10^{-5}$. Momentum spreads in the range $10^{-4}$ to $10^{-5}$ may be reached by classical beam cooling techniques such as electron or laser cooling. For even smaller momentum spreads one may explore the use of "crystallized beams" [48-50]. This is a phenomenon which has been observed for cooled low intensity ion beams, resulting in a transition to an ordered state of the beam with momentum spreads of less than $10^{-6}$. It has yet to be proven whether this technique allows for the extremely high beam intensities required for the neutrino mass measurement proposed here. In addition one needs to separate forward and backward moving electrons with a precision of order $10^{-16}$. In general forward moving electrons will create backward moving electrons by collisions with the walls of the cavern. A suitable experimental configuration to overcome this challenge must be identified.

Given the utmost importance of establishing the absolute value of the neutrino mass in a model independent way, we feel that all possible directions have to be explored. We hope that our work will stimulate more intense investigations on the possibility to use ion beams to pursue this fundamental issue in neutrino physics.

Acknowledgements The authors give thanks for the many useful comments and discussions had with colleagues throughout this work: Jose Bernabeu, Klaus Blaum, Michel Doser, Adrian Fabich, Yuri Litvinov, Mauro Mezzetto, Yu Novikov, Silvia Pascoli, Karsten Riisager and Paul Soler. ML would also like to thank Piero Zucchelli for initially suggesting the idea of using a storage ring for a neutrino mass measurement. CO acknowledges the support of a STFC studentship and CARE, under contract number RII3-CT-2003-0506395, for overseas fieldwork support. CO would also like to thank the ISOLDE group and beta beam group at CERN for their hospitality on a number of occasions. TS acknowledges support by the Transregio Sonderforschungsbereich TR27 "Neutrinos and Beyond" der Deutschen Forschungsgemeinschaft. ML, $\mathrm{CO}$ and TS acknowledge the financial support of the European Community under the European Commission Framework Programme 7 Design Study: EUROnu, Project Number 212372. The EC is not liable for any use that may be made of the information contained herein.

\section{Appendix: Radial distribution}

In this appendix we comment on the radial distribution of the electron events on the back wall of the chamber. One could use a silicon pixel detector which gives position information. One can bin the events as a function of the distance from the beam and perform a fit to the $R$ distribution for each experimental run at given momentum cut $\epsilon$.

Consider an electron with charge $q=1$ and momentum $p_{\perp}$ perpendicular to a magnetic field $B$, which is parallel to the beam axis. The trajectory of the particle will be a helix 


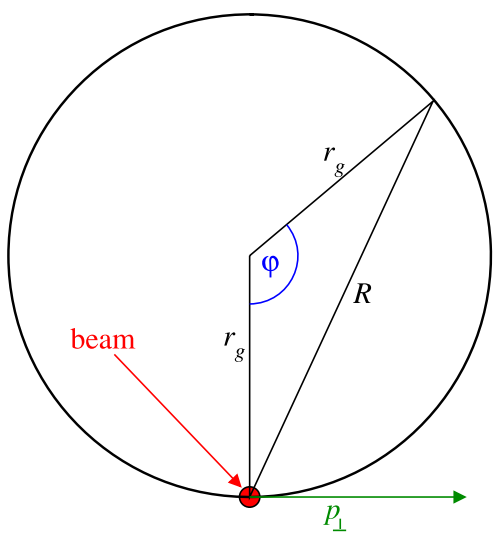

Fig. 7 Trajectory of an electron in the plane perpendicular to the beam

with the maximum distance from the beam axis given by twice the gyro-radius

$R_{\max }=2 r_{g}=\frac{2 p_{\perp}}{q B}$.

The trajectory of the electron in the plane orthogonal to the beam is shown in Fig. 7, where $R$ is the distance from the beam. The electrons will hit the detector with a flat distribution in the angle $\varphi$. We obtain for the differential count rate (compare (16)):

$d N=\frac{1}{2} \frac{d \Gamma}{d E_{\beta}} \frac{p_{\perp}}{p\left(E_{\beta}+m_{e}\right)} d p_{\|} d p_{\perp} \frac{d \varphi}{2 \pi}$.

From Fig. 7 one finds

$R=2 r_{g} \sin \frac{\varphi}{2} \quad$ and $\quad d \varphi=d R \frac{B}{p_{\perp} \sqrt{1-\left(\frac{R B}{2 p_{\perp}}\right)^{2}}}$.

Hence, we obtain

$d N=\frac{B}{4 \pi} \frac{d \Gamma}{d E_{\beta}} \frac{1}{p\left(E_{\beta}+m_{e}\right)} \frac{1}{\sqrt{1-\left(\frac{R B}{2 p_{\perp}}\right)^{2}}} d p_{\|} d p_{\perp} d R$.

Integration of the momentum for a given $\epsilon$ yields the $R$ distribution of the events:

$$
\begin{aligned}
\frac{d N}{d R}= & \frac{B}{4 \pi} \int_{p_{\perp}^{\min }(R)}^{p_{\perp}^{\max }} d p_{\perp} \frac{1}{\sqrt{1-\left(\frac{R B}{2 p_{\perp}}\right)^{2}}} \\
& \times \int_{p_{\max }-\epsilon}^{p_{\|}^{\max }\left(p_{\perp}\right)} d p_{\|} \frac{d \Gamma}{d E_{\beta}} \frac{1}{p\left(E_{\beta}+m_{e}\right)},
\end{aligned}
$$

where $p_{\perp}^{\max }=\sqrt{2 p_{\max } \epsilon-\epsilon^{2}}$ is the maximum perpendicular momentum for given $\epsilon, p_{\perp}^{\min }(R)=B R / 2$ is the minimum perpendicular momentum required to hit at a distance

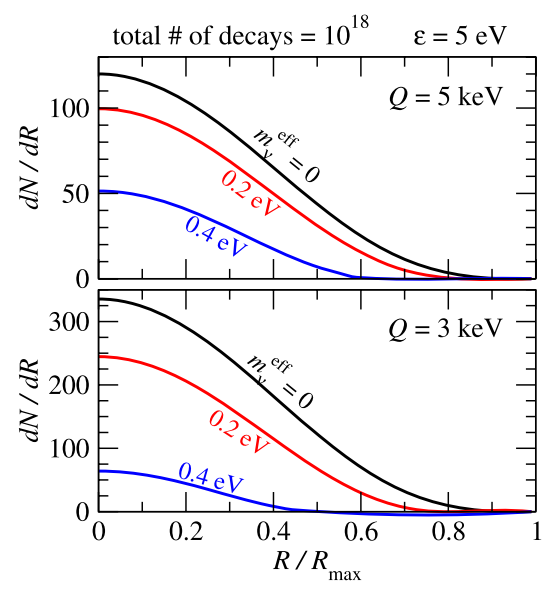

Fig. 8 Number of events as a function of the distance $R$ from the beam axis for $10^{18}$ decays at an ion boost corresponding to $\epsilon=5 \mathrm{eV}$. The distribution is shown for $Q=3$ and $5 \mathrm{keV}$ and for $m_{v}^{\text {eff }}=0,0.2,0.4 \mathrm{eV}$

$R$ from the beam, and $p_{\|}^{\max }\left(p_{\perp}\right)=\sqrt{p_{\max }^{2}-p_{\perp}^{2}}$. Note that integrating (7.5) over $R$ from zero to $R_{\max }$ returns (16).

In Fig. 8 we show some examples for the $R$ distribution. Unfortunately the shape of this distribution carries only limited information on the neutrino mass. The reason is that there is a significant averaging of the endpoint region. Note that the electrons with $p_{\perp}^{\max }$, which have the maximal momentum $p_{\max }$, will hit the wall at all radii from zero to $R_{\max }$. Hence, for small $R$ electrons with rather broad range of momenta contribute. Because of this the $R$ distribution provides only limited additional information, beyond just the decrease of event number with increasing $m_{v}^{\text {eff }}$. This explains the only modest improvement of the sensitivity seen in Fig. 5.

\section{References}

1. Y. Fukuda et al. (Super-Kamiokande Coll.), Phys. Rev. Lett. 81, 1562 (1998). arXiv:hep-ex/9807003

2. M. Ambrosio et al. (MACRO Coll.), Phys. Lett. B 434, 451 (1998). arXiv:hep-ex/9807005

3. M.C. Sanchez et al. (Soudan 2 Coll.), Phys. Rev. D 68, 113004 (2003). arXiv:hep-ex/0307069

4. Y. Ashie et al. (Super-Kamiokande Coll.), Phys. Rev. D 71, 112005 (2005). arXiv:hep-ex/0501064

5. B.T. Cleveland et al., Astrophys. J. 496, 505 (1998)

6. Y. Fukuda et al. (Kamiokande Coll.), Phys. Rev. Lett. 77, 1683 (1996)

7. J.N. Abdurashitov et al. (SAGE Coll.), J. Exp. Theor. Phys. 95, 181 (2002). Z. Eksp. Teor. Fiz. 122, 211 (2002). arXiv:astro-ph/ 0204245

8. W. Hampel et al. (GALLEX Coll.), Phys. Lett. B 447, 127 (1999)

9. T.A. Kirsten (GNO Coll.), Nucl. Phys. Proc. Suppl. 118, 33 (2003)

10. S. Fukuda et al. (Super-Kamiokande Coll.), Phys. Lett. B 539, 179 (2002). arXiv:hep-ex/0205075

11. Q.R. Ahmad et al. (SNO Coll.), Phys. Rev. Lett. 87, 071301 (2001). arXiv:nucl-ex/0106015 
12. Q.R. Ahmad et al. (SNO Coll.), Phys. Rev. Lett. 89, 011301 (2002). arXiv:nucl-ex/0204008

13. Q.R. Ahmad et al. (SNO Coll.), Phys. Rev. Lett. 89, 011302 (2002). arXiv:nucl-ex/0204009

14. S.N. Ahmed et al. (SNO Coll.), Phys. Rev. Lett. 92, 181301 (2004). arXiv:nucl-ex/0309004

15. B. Aharmim et al. (SNO Coll.), Phys. Rev. C 72, 055502 (2005). arXiv:nucl-ex/0502021

16. M. Apollonio et al. (CHOOZ Coll.), Phys. Lett. B 466, 415 (1999). arXiv:hep-ex/9907037

17. M. Apollonio et al. (CHOOZ Coll.), Eur. Phys. J. C 27, 331 (2003). arXiv:hep-ex/0301017.

18. F. Boehm et al., Phys. Rev. Lett. 84, 3764 (2000). arXiv:hep-ex/ 9912050

19. F. Boehm et al., Phys. Rev. D 62, 072002 (2000). arXiv:hep-ex/ 0003022

20. F. Boehm et al., Phys. Rev. Lett. 64, 112001 (2001). arXiv:hep-ex/ 0107009

21. K. Eguchi et al. (KamLAND Coll.), Phys. Rev. Lett. 90, 021802 (2003). arXiv:hep-ex/0212021

22. T. Araki et al. (KamLAND Coll.), Phys. Rev. Lett. 94, 081801 (2005). arXiv:hep-ex/0406035

23. M.H. Ahn et al. (K2K Coll.), Phys. Rev. D 74, 072003 (2006). arXiv:hep-ex/0606032

24. D.G. Michael et al. (MINOS Coll.), Phys. Rev. Lett. 97, 191801 (2006). arXiv:hep-ex/0607088

25. T. Schwetz, M. Tortola, J.W.F. Valle, New J. Phys. 10, 113011 (2008). arXiv:0808.2016 [hep-ph]

26. S.M. Bilenky, C. Giunti, J.A. Grifols, E. Masso, Phys. Rep. 379, 69 (2003). arXiv:hep-ph/0211462

27. F. Feruglio, A. Strumia, F. Vissani, Nucl. Phys. B 637, 345 (2002). Addendum-ibid. B 659, 359 (2003). arXiv:hep-ph/0201291

28. G.L. Fogli, E. Lisi, A. Marrone, A. Melchiorri, A. Palazzo, P. Serra, J. Silk, Phys. Rev. D 70, 113003 (2004). arXiv:hep-ph/ 0408045

29. S. Pascoli, S.T. Petcov, T. Schwetz, Nucl. Phys. B 734, 24 (2006). arXiv:hep-ph/0505226

30. A. de Gouvea, J. Jenkins, arXiv:hep-ph/0507021

31. S. Hannestad, arXiv:0710.1952 [hep-ph]

32. G.L. Fogli et al., Phys. Rev. D 78, 033010 (2008). arXiv:0805. 2517 [hep-ph]

33. W. Maneschg, A. Merle, W. Rodejohann, arXiv:0812.0479 [hep$\mathrm{ph}]$

34. R.N. Mohapatra, J.D. Vergados, Phys. Rev. Lett. 47, 1713 (1981)

35. M. Hirsch, H.V. Klapdor-Kleingrothaus, S.G. Kovalenko, Phys. Rev. D 54, 4207 (1996). arXiv:hep-ph/9603213

36. M. Hirsch, H.V. Klapdor-Kleingrothaus, S.G. Kovalenko, Phys. Rev. D 53, 1329 (1996). arXiv:hep-ph/9502385

37. C. Kraus et al., Eur. Phys. J. C 40, 447 (2005). arXiv: hep-ex/0412056

38. V.M. Lobashev et al., Phys. Lett. B 460, 227 (1999)

39. V.M. Lobashev et al., Nucl. Phys. Proc. Suppl. 91, 280 (2001)

40. A. Osipowicz et al. (KATRIN Coll.), arXiv:hep-ex/0109033

41. A. Monfardini et al., Prog. Part. Nucl. Phys. 57, 68 (2006). Nucl. Instrum. Meth. A 559, 346 (2006). arXiv:hep-ex/0509038
42. V.A. Kostelecky, M. Mewes, Phys. Rev. D 69, 016005 (2004). arXiv:hep-ph/0309025

43. V.A. Kostelecky, M. Mewes, Phys. Rev. D 70, 031902 (2004). arXiv:hep-ph/0308300

44. M. Jerkins, J.R. Klein, J.H. Majors, M.G. Raizen, arXiv:0901. 3111 [nucl-ex]

45. P. Zucchelli, Phys. Lett. B 532, 166 (2002)

46. C. Volpe, J. Phys. G 34, R1 (2007). arXiv:hep-ph/0605033

47. M. Lindroos, M. Mezzetto, Beta Beams (Imperial College Press, London, 2009)

48. F. Diedrich, E. Peik, J.M. Chen, W. Quint, H. Walther, Phys. Rev. Lett. 59, 2931 (1987)

49. R. Bluemel, J.M. Chen, W. Quint, W. Schleich, Nature 334, 309 (1988)

50. U. Schramm, D. Habs, Prog. Part. Nucl. Phys. 53, 583 (2004)

51. E.W. Otten, C. Weinheimer, Rep. Prog. Phys. 71, 086201 (2008)

52. S. Gardner, V. Bernard, U.G. Meissner, Phys. Lett. B 598, 188 (2004). arXiv:hep-ph/0407077

53. S.S. Masood, S. Nasri, J. Schechter, M.A. Tortola, J.W.F. Valle, C. Weinheimer, Phys. Rev. C 76, 045501 (2007). arXiv:0706.0897 [hep-ph]

54. F. Vissani, Nucl. Phys. Proc. Suppl. 100, 273 (2001). arXiv: hep-ph/0012018

55. Y. Farzan, O.L.G. Peres, A.Y. Smirnov, Nucl. Phys. B 612, 59 (2001). arXiv:hep-ph/0105105

56. Y. Farzan, A.Y. Smirnov, Phys. Lett. B 557, 224 (2003). arXiv: hep-ph/0211341

57. S.M. Bilenky, M.D. Mateev, S.T. Petcov, Phys. Lett. B 639, 312 (2006). arXiv:hep-ph/0603178

58. E.W. Otten, J. Bonn, Ch. Weinheimer, Int. J. Mass Spectrom. 251, 173 (2006)

59. Sz. Nagy, T. Fritioff, M. Björkhage, I. Bergström, R. Schuch, Europhys. Lett. 74, 404 (2006)

60. K. Blaum, Phys. Rep. 425, 1 (2006)

61. S.I. Redin (Muon g-2 Collaboration), Prepared for IEEE Particle Accelerator Conference (PAC 99), New York, 29 Mar.-2 Apr. 1999

62. J. Cornell, Caen GANIL-(03/12,rec.Mar.04) 1 CD, 622 p. http://www.eurisol.org

63. H.L. Ravn et al., Nucl. Instrum. Meth. B 88, 441 (1994)

64. C. Volpe, J. Phys. G 30, L1 (2004). arXiv:hep-ph/0303222

65. V.V. Parkhomchuk, in Proceedings of ECOOL 1984, ed. by H. Poth (KfK 3846, Karlsruhe, 1984), p. 71

66. N.S. Dikansky, D.V. Pestrikov, in Proceedings of ECOOL 1984, ed. by H. Poth (KfK 3846, Karlsruhe, 1984), p. 275

67. M. Steck et al., Phys. Rev. Lett. 77, 3803 (1996)

68. H. Danared, A. Källberg, K.-G. Rensfelt, A. Simonsson, Phys. Rev. Lett. 88, 174801 (2002)

69. H. Danared, A. Källberg, A. Simonsson, J. Phys. B At. Mol. Phys. 36, 1003 (2003)

70. I. Ben-Zvi, The ERL high energy cooler for RHIC, in Proceedings EPAC 2006, Edinburgh, Scotland, p. 936

71. U. Schramm et al., Hyperfine Interact. 1, 181 (2005)

72. A. Noda, M. Ikegami, T. Shirai, New J. Phys. 8, 288 (2006)

73. T. Shirai et al., Phys. Rev. Lett. 98, 204801 (2007) 